\title{
Transatlantica
}

Revue d'études américaines. American Studies Journal

\section{La diplomatie électorale de Donald Trump et ses conséquences sur la scène internationale}

Institut du monde anglophone, Paris, 10 janvier 2020

\section{Margaux Magalhaes}

\section{(2) OpenEdition}

\section{Journals}

Édition électronique

URL : https://journals.openedition.org/transatlantica/13826

DOI : $10.4000 /$ transatlantica. 13826

ISSN : 1765-2766

Éditeur

Association française d'Etudes Américaines (AFEA)

Référence électronique

Margaux Magalhaes, «La diplomatie électorale de Donald Trump et ses conséquences sur la scène internationale », Transatlantica [En ligne], 1 | 2019, mis en ligne le 01 juin 2020, consulté le 03 février 2023. URL : http://journals.openedition.org/transatlantica/13826 ; DOI : https://doi.org/10.4000/ transatlantica.13826

Ce document a été généré automatiquement le 3 février 2023

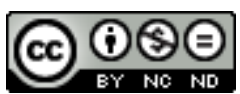

Creative Commons - Attribution - Pas d'Utilisation Commerciale - Pas de Modification 4.0 International - CC BY-NC-ND 4.0

https://creativecommons.org/licenses/by-nc-nd/4.0/ 


\title{
La diplomatie électorale de Donald Trump et ses conséquences sur la scène internationale
}

Institut du monde anglophone, Paris, 10 janvier 2020

\author{
Margaux Magalhaes
}

1 À l'occasion de cette journée d'étude, co-organisée par l'Institut Open Diplomacy et le Center for Research in the English-speaking World (CREW) de l'université Sorbonne Nouvelle-Paris 3, les intervenants se sont attachés à analyser les défis posés par la présidence Trump sur la scène internationale, en accordant une place centrale au poids des déterminants intérieurs. Il est vrai que lorsque l'on s'intéresse à la politique étrangère d'une démocratie, on ne peut faire abstraction des dynamiques intérieures, a fortiori dans le cadre d'une conférence sur les États-Unis en 2020, année électorale se déroulant sur fond de procès de destitution du locataire de la Maison-Blanche. La journée d'étude avait donc pour objectif une meilleure compréhension de la politique étrangère menée par la principale puissance économique et militaire mondiale, dont les décisions ne cessent de surprendre les Européens par leur imprévisibilité et leurs incohérences supposées.

\section{Masterclass}

2 La matinée a débuté par l'intervention percutante de Nathalie Loiseau, députée européenne, présidente de la sous-commission Défense et ancienne ministre française chargée des affaires européennes. Elle précise tout d'abord que deux écueils sont à éviter lorsque l'on évoque la diplomatie de Trump : la caricature et la banalisation. Elle souligne en outre qu'il ne faudrait pas donner aux actions du président une valeur ajoutée là où elles n'auraient été que des paris. Car, pour Nathalie Loiseau, Trump est bien avant tout un parieur. Elle considère dès lors que ses choix politiques ne sont pas imprévisibles, en ce qu'il fait ce qu'il a toujours dit qu'il ferait, à savoir poursuivre sa politique d'America First. Pour lui, il n'y aurait pas d'alliés mais uniquement des clients 
ou des concurrents, de sorte que les Européens sont traités au même titre que de simples partenaires de circonstance. Bien que Trump soit le premier président américain à afficher ouvertement son hostilité au projet européen, une majorité d'Européens vivrait dans la nostalgie d'une relation transatlantique privilégiée - en particulier en Europe de l'Est, toujours hantée par la menace russe. C'est d'ailleurs la résurgence de cette dernière qui amènerait la région à participer aux efforts de défense européenne portée par la France. Elle évoque ensuite le sujet brûlant de l'Iran. Soulignant combien le régime des mollahs est détesté aux États-Unis, elle précise la difficulté que pose un retour à un équilibre sur la question du nucléaire, tant il fut laborieux d'amener la superpuissance à conclure l'accord de Vienne (ce qui a nécessité près de dix ans). Elle précise enfin que les États-Unis effectuent un pivot vers le Pacifique au détriment de l'Europe et du Moyen-Orient au nom d'arguments d'ordre économique. Ce qui les empêcherait d'aller plus loin en 2020 serait leur attachement à Israël, la relation américano-israélienne n'ayant jamais été aussi forte que sous l'administration Trump.

\section{Les déterminants électoraux d'une diplomatie particulière}

3 Le premier panel s'ouvre ensuite sur la thématique « les déterminants électoraux d'une diplomatie particulière ", lors duquel Alexis Garrati (Euler Hermes), Maya Kandel (CREW), Laurence Nardon (IFRI), Nicolas Tenzer (CERAP) et Christine Zumello (CREW) s'expriment.

4 La première intervenante, Maya Kandel, explique que la politique étrangère de Trump se caractérise par un nationalisme et une politique dite jacksonienne aux forts accents économiques ${ }^{1}$. C'est cette orientation qui expliquerait l'intervention américaine contre Soleimani : Trump chercherait à parler à sa base. Cette dernière serait sensible à sa présentation de l'élimination du haut dignitaire iranien comme celle d'un terroriste responsable de la mort d'Américains, discours séduisant en outre les vétérans et ceux qui souhaitent protéger l'Amérique sans pour autant vouloir une guerre. Elle rappelle ensuite que le choix de l'élimination du général iranien ne fait pas consensus, ni auprès des Républicains ni auprès des Démocrates, certains voulant abandonner les politiques interventionnistes. Elle souligne également l'importance que revêt l'obsession du président américain envers Obama, laquelle conditionne, en partie, ses choix de politique étrangère, ainsi que l'illustre le cas Soleimani. Trump ne se vante-t-il pas d'être parvenu à éliminer deux terroristes (al-Baghdadi et Soleimani) quand Obama, lui, ne peut se prévaloir que de Ben Laden? Enfin, elle précise que les Américains ne votent généralement pas sur des questions de politique étrangère, à l'exception des problématiques migratoires ou économiques.

5 Nicolas Tenzer, lui, souligne tout d'abord la continuité entre la politique étrangère menée par Obama et celle de Trump, qui est celle du désengagement. Mais, précise-t-il, la base républicaine adopterait, elle, une position contradictoire : elle serait à la fois attachée à une image de puissance et d'une Amérique imposant sa norme, mais refuserait l'intervention armée ou les engagements au nom de la propagation des valeurs "universelles", c'est-à-dire libérales. D'ailleurs, les Américains dans leur ensemble ne souhaitent pas voir leurs impôts augmenter pour financer l'interventionnisme; il y aurait dès lors un fond isolationniste très marqué aux Etats- 
Unis. Cependant, les Républicains de la vieille école estiment à l'inverse que l'Amérique doit s'engager pour propager ses valeurs. N. Tenzer poursuit son analyse en précisant que Trump n'a aucune stratégie, ni en Syrie, ni en Iran, ni ailleurs. Contrairement à Nathalie Loiseau, il estime que le président américain ne dispose pas d'une vision cohérente. Sa diplomatie, ce serait simplement celle du renoncement au leadership américain. Tout comme Maya Kandel, il explique pour conclure que la politique étrangère américaine n'est que peu conditionnée par un facteur électoral, les Américains ne votant pas sur des questions d'ordre international mais plutôt sur des questions nationales (éducation, avortement, etc.).

6 L'intervention d'Alexis Garrati, quant à elle, offre un éclairage intéressant sur la dimension économique de la politique étrangère de Trump. Selon lui, le président américain disposerait d'une véritable stratégie économique, symbolisée par l'America First et privilégiant la création d'emplois dans son pays, si bien que les États-Unis connaissent aujourd'hui le plein-emploi ${ }^{2}$. Cette politique ne serait toutefois pas sans implications sur la scène internationale: elle contribuerait même au désordre économique mondial. Par conséquent, cette posture aura en réalité des répercussions sur les États-Unis eux-mêmes : le choc extérieur et la dégradation de la conjoncture internationale créés par Trump entraineront une diminution des investissements extérieurs, ce qui ne sera pas sans effet sur la croissance américaine et, par voie de conséquence, sur leur taux d'emploi. La dette pourrait également pâtir de cette situation.

7 C'est ensuite à Christine Zumello de prendre la parole. Elle explique tout d'abord que ses bonnes performances économiques et le plein-emploi qui en découle valent à Trump un soutien constant. S'il a été président, précise-t-elle, c'est en outre grâce à une stratégie électorale dénonçant les malversations et la corruption prêtées à Washington : se présentant comme étranger à cet establishment, il porterait la voix des Américains oubliés, selon une rhétorique particulièrement populaire dans les États pivots du Michigan ou du Wisconsin.

8 Laurence Nardon clôt ce panel en précisant que la dégradation du discours public aux États-Unis n'est pas le simple fait de Trump mais relève de causes plus anciennes, alimentées par la télévision câblée et l'usage généralisé d'internet. Afin d'accroître leur audience - et donc leurs recettes-, les médias radicaliseraient leur discours, contribuant à polariser la scène politique américaine. Trump ne fait donc qu'aggraver cette tendance antérieure. D'ailleurs ses incartades séduiraient particulièrement sa base électorale, populiste et anti-élite, qui y voit la preuve que Trump est antisystème et qu'il comprend ses préoccupations. Dans ces conditions, comment une puissance si polarisée pourrait-elle être un allié fiable ? s'interroge-t-elle.

\section{Les réseaux exogènes d'un président en campagne}

9 Dans ce deuxième panel s'expriment Cyrille Bret (Sciences Po), Jeffrey Hawkins (IRIS), Anne Kraatz (École Pratique des Hautes Études), Sébastien Maillard (Institut Jacques Delors) et Pauline Schnapper (CREW) sur «les réseaux exogènes d'un président en campagne ».

Cyrille Bret, tout d'abord, souligne la continuité des politiques menées par Obama et Trump à l'égard des Européens (caractérisées par leur volonté de désengagement et de partage du fardeau). Il explique ensuite que Trump ne serait pas original, si ce n'est 
dans son style qui se veut provocateur. Il souligne néanmoins son absence de cohérence idéologique à l'international, rappelant que sa priorité reste la politique intérieure. Il précise enfin que le président américain rompt avec le wilsonnisme pour se rapprocher de la vision de Théodore Roosevelt, d'après qui tout accord produit nécessairement un gagnant et un perdant, réfutant donc la possibilité de rapports gagnant-gagnant. Pour Trump, le multilatéralisme serait la revanche du faible.

11 L'intervention de Sébastien Maillard s'articule elle aussi principalement autour de la relation transatlantique. Il explique tout d'abord que face à Trump, l'Union européenne peine à parler d'une seule voix, en raison d'intérêts divergents. Pour l'Allemagne, par exemple, le lien transatlantique serait presque une raison d'État; il lui serait donc difficile d'imaginer que celui-ci puisse s'éloigner. Néanmoins, la politique de Trump, conclut-il, obligerait les dirigeants du Vieux Continent à accélérer le projet européen.

Pauline Schnapper, quant à elle, analyse avant tout la « relation spéciale » entre les États-Unis et la Grande-Bretagne. Elle revient tout d'abord sur le peu d'affinité de Trump à l'égard du projet européen: il serait le premier président à témoigner ouvertement son hostilité, ainsi qu'il l'a montré avec le Brexit. Dans un geste sans précédent, Trump n'a pas hésité à s'immiscer directement dans le processus électoral d'un pays allié, à prendre position en faveur du départ du Royaume-Uni, et à critiquer une Theresa May (et avec elle, un gouvernement ami) qu'il cherchait à affaiblir.

Jeffrey Hawkins, lui, s'intéresse à la primauté accordée par Trump à la politique intérieure, ce qui le conduirait à vouloir libérer l'Amérique de la contrainte du leadership et de la propagation de ses valeurs, et à adopter des mesures protectionnistes et populistes. C'est un président qui ne serait pas contraint par une idéologie sur la scène internationale, ce qui expliquerait des décisions pouvant froisser l'establishment (sur la Russie, les Kurdes, la Corée du Nord, etc.). Il n'hésiterait alors pas à trahir ses alliés en maintenant des relations très équivoques avec l'adversaire. Il en conclut que le système construit après la Seconde Guerre mondiale serait en train de s'effondrer, même s'il rappelle que la vision de Trump serait minoritaire aux États-Unis.

Anne Kraatz insiste, pour sa part, sur le vote des vétérans qui se rallieraient à un Trump promettant de rapatrier les troupes. D'ailleurs, analyse-t-elle, si les Irakiens souhaitaient effectivement chasser les troupes américaines présentes dans leur pays suite à l'assassinat de Soleimani, cela se révélerait bénéfique électoralement à Trump. Elle rejoint les autres intervenants en précisant que la politique étrangère de Trump s'inscrit dans la continuité de celle menée par Obama, tous deux considérant la chine et la Russie comme plus importantes pour la stabilité et la prospérité américaine que l'Europe. La nouveauté, ce serait son style populiste.

\section{"The Art of the Deal », droit international et rapports de puissance}

15 Dans ce troisième panel sur " "The Art of the Deal", droit international et rapports de puissance ", se succèdent les interventions d'Annick Cizel (CREW), François Clemenceau (le JDD), Anne Deysine (Université Paris Nanterre), Michel Duclos (Institut Montaigne) et Denis Ferrand (Rexecode).

16 Annick Cizel revient tout d'abord sur les lignes de continuité entre Obama et Trump. Elle explique que l'évolution de la mondialisation a multiplié les acteurs, imposant aux 
leaders américains une redéfinition de leur leadership par défaut, dans un monde où émergent des puissances concurrentes. C'est ainsi que dans la Stratégie de sécurité nationale de 2017, la Russie et la Chine figurent au sommet de ses préoccupations. Le réalisme de Trump serait donc palpable et expliquerait sa stratégie globale de retrenchment, héritée d'Obama. Sous Trump, la diplomatie serait en outre rendue futile, du fait notamment de dangers devenus transnationaux : face à des forces comme alQaeda ou Daech par exemple, le rapport de force prévaut puisque les négociations ne sont pas possibles. Qu'importe l'administration, une nouvelle donne internationale dictera les choix de Washington, conclut-elle. Pour l'heure, la politique de Trump pourrait être caractérisée par une rivalité agressive à l'endroit de l'Union européenne, par son anti-multilatéralisme et par des prises de positions nationalistes et patriotiques.

François Clemenceau, lui, affirme que depuis 2017 la politique étrangère de l'Amérique se réduit tout entière à la personne de Trump. Comprendre sa personnalité et son parcours seraient donc essentiel: c'est un président qui serait ignorant du monde, de la géopolitique ou de la stratégie, d'où des politiques incohérentes et imprévisibles. Il ne disposerait pas non plus de vision: ce serait un président sans trajectoire. En outre, il serait un président cynique, basant ses décisions sur des gains à court terme, notamment électoraux.

Anne Deysine souligne également le rejet absolu du multilatéralisme de Trump, qui préfère les relations bilatérales. Ce choix de l'Amérique s'accompagne, selon elle, d'une perte de son leadership et de son soft power. En parallèle, s'inscrivant dans la continuité de Clinton, la diplomatie américaine est démantelée, le président ne pourvoyant pas à de nombreux postes et diminuant mécaniquement l'influence américaine sur la scène internationale. Elle rappelle enfin la préférence de Trump pour la politique des sanctions tous azimuts, ce qui choque notamment en Europe.

Pour Michel Duclos, les Américains auraient toujours été isolationnistes ; la nouveauté serait sous Trump la volonté de remettre en cause les structures de coopération. Il explique ensuite que la politique transactionnelle de Trump ne lui a apporté que peu de succès : quel accord positif a-t-il passé ? s'interroge-t-il.

Denis Ferrand, enfin, précise que la diplomatie de Trump est en réalité anti-électorale, dans le sens où ses politiques économiques vont se retourner contre la superpuissance. $\mathrm{Du}$ point de vue des flux financiers, sa politique aurait été un échec. Il évoque également les problèmes inhérents à l'OMC, notamment l'hégémonie chinoise qui s'est instituée au détriment de la superpuissance mais aussi les litiges (tels le refus des ÉtatsUnis d'y nommer un juge). Il conclut son analyse en précisant que Trump a remis en cause la diplomatie économique telle que pratiquée jusqu'alors par la superpuissance.

\section{Sécurité globale : le grand jeu de Trump}

21 Dans ce dernier panel étudiant la "sécurité globale: le grand jeu de Trump", s'expriment Martin Quencez (German Marshall Fund), Guillaume Lasconjarias (IFRI), Benoît Pelopidas (Chaire Nuclear Knowledges, CERI / Sciences Po), Marc Sémo (Le Monde) et Clément Therme (Nuclear Knowledges, CERI / Sciences Po).

Martin Quencez évoque dans son intervention la relation transatlantique: les divisions européennes seraient responsables, avant toute chose, des problèmes qui la 
traversent. Il montre tout d'abord que l'Allemagne prône la patience face à Trump : pour elle, il n'y aurait pas d'alternative à la relation transatlantique. Elle n'est donc guère favorable à l'autonomie stratégique prônée par la France. À l'instar des autres intervenants, il souligne la continuité de la politique d'obama et Trump envers les Européens, à ceci de différent qu'Obama voulait réduire la présence américaine en s'appuyant sur des partenariats alors que Trump n'a guère d'appétence pour les alliances. Il précise enfin que le fossé technologique entre les deux rives de l'Atlantique continuera à empoisonner cette relation.

Guillaume Lasconjarias, à son tour, précise que Trump n'aime pas les alliances contraignantes, préférant des relations bilatérales à une approche multilatérale, alimentant par là la méfiance de ses alliés. Selon lui, pour le président américain, les alliances doivent rapporter des dividendes immédiats à l'Amérique.

Marc Sémo fait également un lien entre les politiques d'Obama et de Trump, en Irak et en Syrie, ou encore dans leur pivot vers l'Asie et leur recours aux assassinats ciblés. Il revient sur la qualification de l'OTAN comme « obsolète » selon Trump, puis en «état de mort cérébrale » selon Macron, estimant que les deux dirigeants ont établi un bon diagnostic. Enfin, il explique que l'imprévisibilité de Trump représente un vrai problème pour les Européens, qui ne peuvent plus compter sur l'Amérique.

Selon Benoît Pelopidas, ce qui est marquant chez Trump, c'est son manque de sérieux vis-à-vis de la question nucléaire. N’a-t-il pas naïvement déclaré : « si on en a, pourquoi ne l'utilise-t-on pas ?» Pour lui, la diplomatie nucléaire de Trump est avant tout interpersonnelle, ainsi que l'illustre la Corée du Nord. Il s'interroge également sur la sortie du traité INF : se dirige-t-on vers une escalade et une sur-réaction?

Enfin, pour conclure ce panel, Clément Therme évoque la crise entre les États-Unis et l'Iran, expliquant qu'aucune des deux parties ne souhaite la guerre, et surtout pas l'Iran $\mathrm{du}$ fait d'un rapport de force asymétrique. Il souligne ensuite la tendance isolationniste des États-Unis et revient sur les différends transatlantiques. Il explique que l'Europe se dresse parfois contre l'Amérique mais, faute d'autonomie, doit s'allier à elle, ainsi que l'illustre le cas iranien : en dépit de la contestation des Européens suite au retrait de la superpuissance de l'accord de Vienne, il n'y a plus d'entreprises françaises en Iran.

\section{Masterclass}

27 La journée s'achève sur l'intervention de Denis MacShane, ancien ministre d'État en charge des Affaires étrangères du Royaume-Uni. Il commence en faisant un parallèle entre Trump et le Brexit, tous deux survenus la même année : ils sont l'expression du populisme, du nationalisme et du rejet de l'ordre établi. Il évoque ensuite l'impact que pourrait avoir le Brexit sur le projet européen, avant d'analyser les attitudes des dirigeants autoritaires envers l'état de droit ou les libertés d'expression.

$\mathrm{Au}$ total, la journée d'étude aura offert un panorama satisfaisant de la politique étrangère de Trump, même si on pourrait regretter qu'une approche euro-centrée ait été privilégiée, et que le Moyen-Orient et surtout l'Asie et l'Amérique Latine, dont l'importance ne cesse de croître pour la superpuissance (tant pour des raisons intérieures qu'extérieures), n'aient pas été davantage étudiés. 


\section{NOTES}

1. Le jacksonisme fait référence à la politique populiste et nationaliste poursuivie au XIX ${ }^{\mathrm{e}}$ siècle par le président Jackson. Pour plus de précisions, se reporter à l'article de Walter Russell Mead, "The Jacksonian Tradition: And American Foreign Policy», The National Interest, $\mathrm{n}^{\circ} 58$, hiver 1999-2000, p. 5-29.

2. Rappelons que cette journée d'étude s'est tenue avant le début de la pandémie de Covid-19.

INDEX

Thèmes : Actualité de la recherche

\section{AUTEUR}

\section{MARGAUX MAGALHAES}

Sciences Po Lille 